\title{
Embedded bleeding detector into a PMMA applicator for electron intraoperative radiotherapy
}

\author{
Rafael Garcia-Gil ${ }^{\text {a }}$, Silvia Casans ${ }^{\text {a }}$, A. Edith Navarro ${ }^{\text {a }}$, Antonio-José García-Sánchez ${ }^{\text {, }}$, \\ Juan J. Rovira-Escutia ${ }^{c}$, Daniel Garcia-Costa ${ }^{\mathrm{d}}$, Enrique Sanchis-Sánchez ${ }^{\mathrm{e}, *}$, \\ Ignacio Pérez-Calatayud ${ }^{\mathrm{f}}$, José Pérez-Calatayud ${ }^{\text {g, h, }}{ }^{\text {, }}$, Enrique Sanchis ${ }^{\text {a, }}$ i

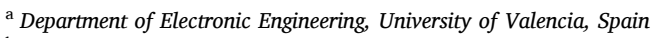 \\ b PTW Dosimetría Iberia SLU, Valencia, Spain

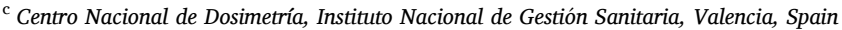 \\ ${ }^{\mathrm{d}}$ Department of Informatics, University of Valencia, Spain \\ ${ }^{\mathrm{e}}$ Department of Physical Therapy, University of Valencia, Spain \\ ${ }^{\mathrm{f}}$ Aplicaciones Tecnológicas S.A., Valencia, Spain \\ ${ }^{g}$ Department of Radiation Oncology, La Fe Hospital, Valencia, Spain \\ ${ }^{\mathrm{h}}$ Department of Radiation Oncology, Clínica Benidorm, Alicante, Spain \\ ${ }^{\text {i }}$ IRIMED Joint Research Unit IIS La Fe - University of Valencia, Spain
}

\section{A R T I C L E I N F O}

\section{Keywords:}

Intraoperative radiotherapy

Bleeding detector

Capacitive sensor

\begin{abstract}
A B S T R A C T
Purpose: The aim of this work is to present a ready to industrialize low-cost and easy-to-install bleeding detector for use in intraoperative electron radiation therapy (IOERT). The detector works in stand-alone mode and is embedded into a translucent polymethylmethacrylate (PMMA) applicator avoiding any contact with the patient, which represent a novelty compared to previous designs. The use of this detector will prevent dose misadministration during irradiation in the event of accumulation of fluids in the applicator.

Methods: The detector is based on capacitive sensor and wireless power-supply electronics. Both sensor and electronics have been embedded in the applicator, so that any contact with the patient would be avoided. Since access to the tumor can be done through different trajectories, the detector has been calibrated for different tilting angles. Results: The result of the calibration provides us with a fit curve that allows the interpolation of the results at any angle. Comparison of estimated fluid height vs real height gives an error of $1 \mathrm{~mm}$ for tilting angles less than $10^{\circ}$ and $2 \mathrm{~mm}$ for tilting angles greater than $15^{\circ}$. This accuracy is better than the one required by clinic. Conclusions: The performance of the bleeding detector was evaluated in situ. No interference was observed between the detector and the beam. In addition, a user-friendly mobile application has been developed to help the surgical team making decisions before and during irradiation. The measurement provided by the mobile application was stable during the irradiation process.
\end{abstract}

\section{Introduction}

Intraoperative radiation electron radiotherapy (IOERT) is a technique that is currently on the rise [1]. This is due to the development of portable electron linear accelerators that are equipped with adequate self-shielding that allows their use in conventional operating rooms. IEORT is currently applied to a wide variety of specific diseases in exclusive mode or combined with external radiotherapy, becoming a relevant option within the modalities of the state of the art in radiotherapy [2-6].
Lately, electron therapy and in particular IOERT has created enormous expectations in the radiation therapy community given the imminent availability of portable electron devices that allow FLASHtype treatments [7]. The FLASH effect in radiotherapy is a radiobiological effect characterized by a loss of radiobiological effectiveness on healthy tissue and an unaltered therapeutic efficacy on tumour tissue. This effect is obtained by delivering the dose with an extremely high dose rate (above $40 \mathrm{~Gy} / \mathrm{s}$ ) [8].

In IOERT, one of the risks that may cause dose misadministration events is the potential bleeding in the tumour bed during the irradiation

\footnotetext{
* Corresponding author at: Department of Physical Therapy, University of Valencia, Spain.

E-mail addresses: enrique.sanchis-sanchez@uv.es (E. Sanchis-Sánchez), enrique.sanchis@uv.es (E. Sanchis).
} 
[3]. This is especially critical in high-vascularized scenarios or in presence of organic fluids. In fact, it was evidenced in the in vivo experimental study of López-Tarjuelo et al. [9] in which they faced with significant deviations due to the blood presence.

To avoid this important risk, the existence of detectors that can show this circumstance is very useful in the clinic. A capacitive based bleeding detector was proposed by Sanchis et al. and Garcia-Gil et al. [10-12] for translucent polymethyl methacrylate applicators (PMMA), as used in the LIAC HWL Mobile linac from Sordina IORT Technologies [13]. A simple capacitive-based detector using a semi-circular geometry was presented in [10]. In this detector, the measurement process was based on repetitive charge-discharge cycles of the capacitor through a resistor. A capacitive-based detector using a geometry based on two capacitive plates each one formed by a set of strips was presented in [11]. In this contribution, improvements were focused on minimizing the impact of the detector on the visual through the plastic applicators as well as avoiding the asymmetry in the detection capability when the applicator is tilted. Finally, the whole electronic design of the bleeding detector presented in [11] was analysed in [12].

In order to use the proposed detector in the clinic, the main problem to be solved is to ensure that both the sensor and the electronics must be embedded in the applicator, thus avoiding direct contact with the patient. This implies that both the data transmission and the charging of the power supply must be wireless. Other goal to achieve is the fluid level detection in any of the possible inclinations of the applicator during the irradiation process.

The aim of this work is to develop a low-cost and easy to install bleeding detector, based on a previously designed prototype [12], to monitor the possible presence of bleeding or organic fluid inside a translucent polymethyl methacrylate applicator (PMMA), used in IOERT.

We present the technical details for the detector development. On the one hand, those referring to the embedding process in the applicator. On the other hand, the electronics, including the AC bridge, the conditioning circuit and the read-out, all of them wirelessly powered. In addition, so that the surgery team can easily verify the level of fluid that has been accumulated in the applicator, a very intuitive and easy-to-use mobile application has been developed.

The performance of the bleeding detector has been evaluated under irradiation in order to detect possible interferences with the beam.

\section{Matherial and methods}

\subsection{Mobile linac}

The LIAC HWL Mobile linac [13] is provided with electron energies of $6,8,10$ and $12 \mathrm{MeV}$. It uses PMMA applicators in order to allow direct visualization of the tumour bed. Applicators have diameters ranging from $3 \mathrm{~cm}$ to $12 \mathrm{~cm}$ with terminal bevel angles ranging from $0^{\circ}$ to $45^{\circ}$. The applicators are composed of two pieces: the upper one attached to the linac head and a second one in contact with the patient. The connection of the two-applicator pieces is done using toggle latches by the so-called "hard-docking". The radiation treatment for typical doses up to $20 \mathrm{~Gy}$ is completed in less than two minutes. The dose per pulse with the $10 \mathrm{~cm}$ in diameter applicator can vary, approximately, from 1 to $8 \mathrm{cGy} / \mathrm{p}$ and the corresponding dose rate can be set from 10 to $30 \mathrm{~Gy} /$ $\min$ [13].

The equipment has been designed to reduce the peripheral stray radiation (SR) so that the linac can be used in a conventional (unshielded) operating room. This has been achieved by reducing the interaction between the accelerated electron beam and any metallic element by using an optical system. In this way, it has been possible to minimize the global SR, obtaining $0.2 \mu \mathrm{Sv} / \mathrm{Gy}$ in the patient plane at a distance of $3 \mathrm{~m}$ from the target [13]. This reduction in SR allows the workload to be maximized.

\subsection{Read-out circuit}

The block diagram of the bleeding detector system used in this paper is shown in Fig. 1 . It is based on a capacitive sensor ( $C_{x}$ in Fig. 1$)$ as described in $[10,11]$ and an electronic conditioning circuit based on AC bridge as described in [12]. It also includes a wireless power supply necessary to charge a supercapacitor (SC in Fig. 1).

The sensor geometry is described in [11]. It is based on two capacitive plates, each one formed by a set of strips (Fig. 2a). The strips of each plate are interspersed with each other in such a way that it reduces the asymmetry effects in the distribution of the fluid to be detected, regardless the different orientations of the applicators used when it is tilted. Besides, it allows the surgery team to have a high lateral visibility through the applicator. This sensor was implemented using a $500 \mu \mathrm{m}$ thick kapton strip that has been embedded and sealed inside the applicator. The detail of the sensor once placed inside the applicator is shown in Fig. 2b.

The wireless power supply of the entire system has been implemented using a resonant circuit, consisting of two antennas (transmitter and receiver) and the capacitors $C_{e}$ and $C_{r}$ (Fig. 1), as presented in [12]. The receiving antenna must be embedded in the applicator itself. A helical ring-shaped design implemented on a $500 \mu \mathrm{m}$ thick kapton flexible printed circuit has been used. Fig. 3a shows the detail of the antenna once sealed in the upper cap of the applicator. A transmitting antenna, located outside the applicator, has been designed to obtain the maximum coupling between them. The transmission stage is based on Shenzhen Core Ketai Electronics Co., Ltd commercial wireless charger module, powered by a commercial $12 \mathrm{~V} / 500 \mathrm{~mA}$ external charger.

The receiving stage allows charging the supercapacitor SC (Fig. 1). The SC has been designed for an autonomy of $80 \mathrm{~min}$, greather than the minimum required by clinic ( $30 \mathrm{~min}$ ). A step-up regulator followed by a polarity inverter is required to obtain the symmetrical $\pm 5 \mathrm{~V}$ supply needed by the measurement circuit. The power board has been implemented in a $500 \mu \mathrm{m}$ thick kapton flexible printed circuit, as shown in Fig. 3b.

In order to measure the very small capacitance variations of the proposed level transducer (in the range of $0.1 \mathrm{pF}$ ) an AC-Bridge circuit as explained in [12] has been used (Fig. 1). The AC source is generated by means of a Wien bridge oscillator. The AC-bridge is formed of a reference resistor-capacitance $\left(R_{0}-C_{o}\right)$, the capacitor sensor $\left(C_{x}\right)$ and a potentiometer $\left(R_{x}\right)$ for bridge balancing. Because the capacitance changes to be measured are very small (less than $0.1 \mathrm{pF}$ ) a large amplification (IA) is necessary. The output of the AC-Bridge $\left(v_{d}\right)$ is connected to the input of the instrumentation amplifier (U1), whose gain is adjustable via $R_{g}$. The IA output is rectified and filtered to obtain a DC voltage $\left(V_{o}\right)$ which is proportional to the fluid level inside the applicator. The conditioning board has also been implemented with $500 \mu \mathrm{m}$ thick kapton flexible printed circuit and is shown in Fig. 3c.

The microcontroller acquires $V_{o}$ and transmits it via Bluetooth to a mobile phone (Fig. 1). For simplicity, an Arduino Nano 33 BLE (Bluetooth Low Energy, 12 bits ADC) has been used. The microcontroller board includes an accelerometer to measure the applicator tilting angle. Likewise, it measures the supercapacitor charge level.

In order to integrate the read-out circuit in the applicator itself, a PMMA tube has been manufactured as a replica of the applicator used by the linac. This tube has been emptied at its head to embed the sensor and the connection cable between the sensor and the conditioning circuit. As shown in Fig. 4a, the upper part has been emptied in order to accommodate all the necessary circuitry: wireless power supply, supercapacitor-based energy storage system, conditioning circuit and microcontroller board. The placement of all the components that make up the system is shown in Fig. $4 \mathrm{~b}$ and the sealed tube with all the circuitry embedded in it is shown in Fig. 4c (henceforth instrumented applicator). 


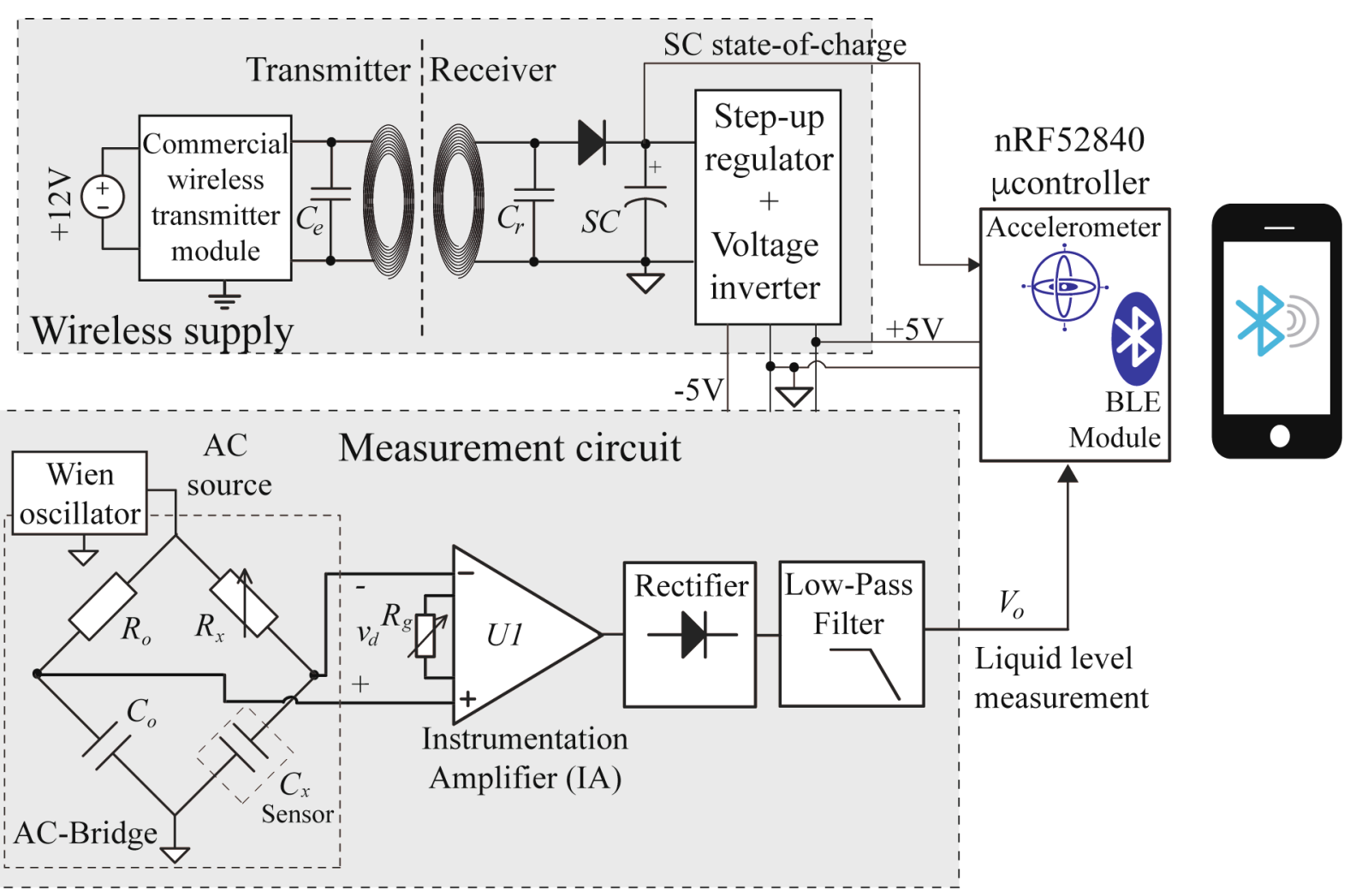

Fig. 1. Bleeding detection system block diagram.

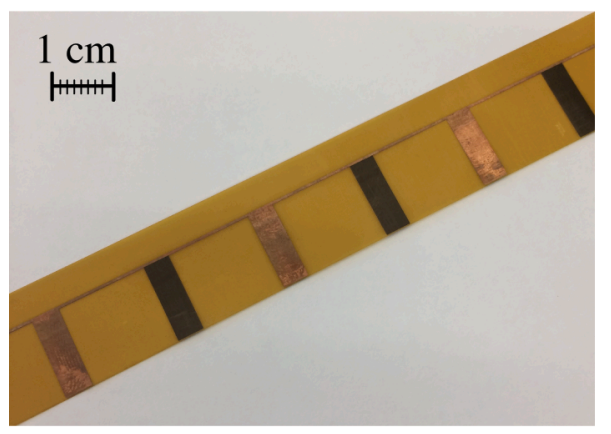

a)

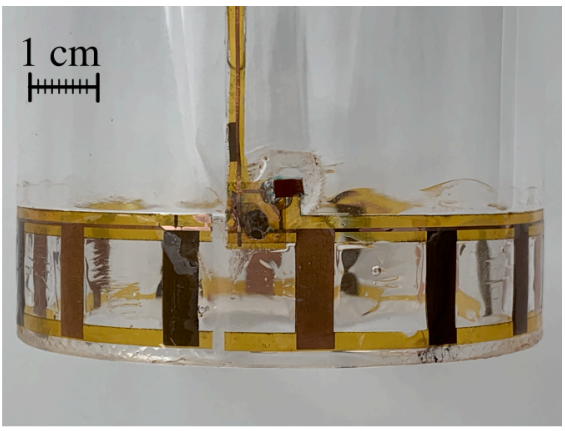

b)

Fig. 2. Capacitive sensor. (a) Sensor implemented in a kapton printed circuit board; (b) Sensor embedded into a 7 cm applicator.

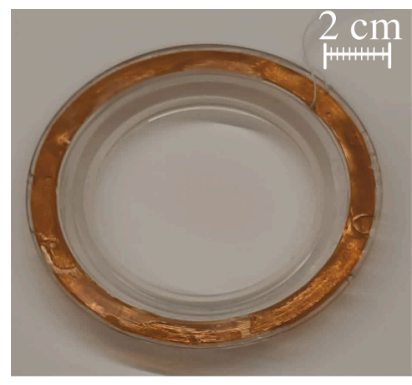

a)

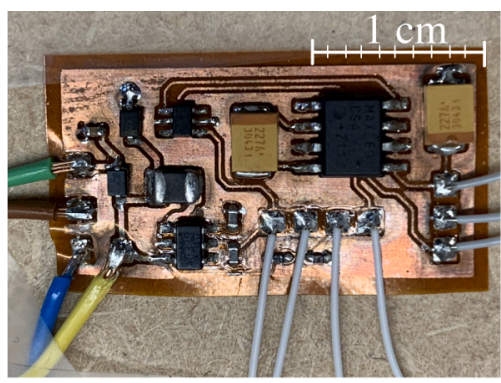

b)

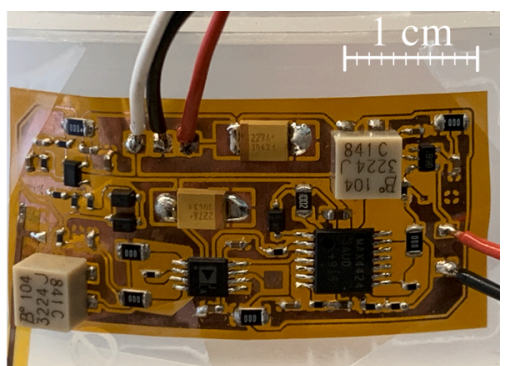

c)

Fig. 3. Details of the implemented electronics. (a) Receiving antenna; (b) Wireless power supply circuit; (c) Measurement circuit. 


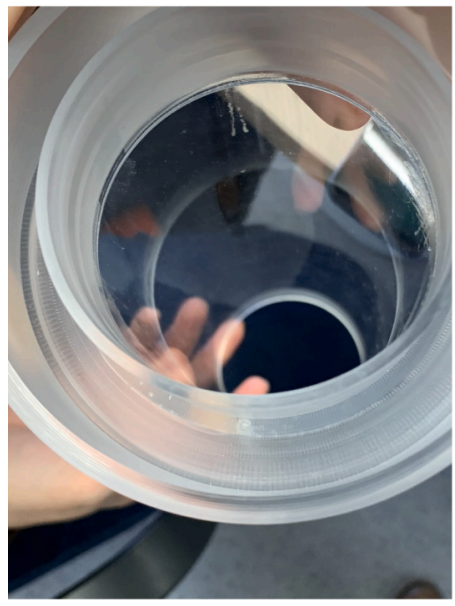

a)

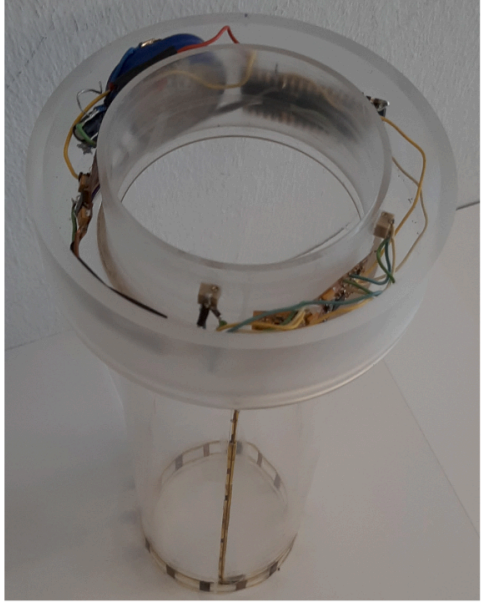

b)

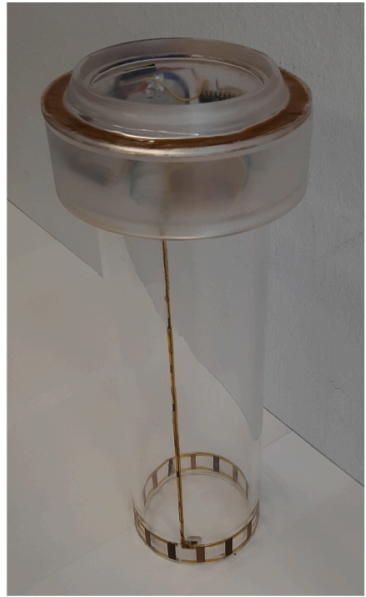

c)

Fig. 4. Embedding process of the electronics into the $7 \mathrm{~cm}$ manufactured tube. (a) Emptied; (b) Positioning; (c) Sealed.

\subsection{Monitoring}

A mobile application has been developed for the surgery team to visualize the level of fluid accumulated in the applicator before the irradiation treatment. The mobile application has been developed using the Apache Cordova open-source platform. This application consists of two views (Fig. 5). The main view (Fig. 5a) shows a graphical representation of the applicator indicating: i) height reached by the fluid; ii) applicator diameter; iii) tilting angle; iv) start measurement button. A traffic light-based fluid level indication (green, yellow, and red) is included in this view to make the surgery team's decision easier. The configuration view (Fig. 5b) allows to toggle between the different applicator diameters available, change the sensitivity according to the calibration results and define the intervals of the aforementioned traffic

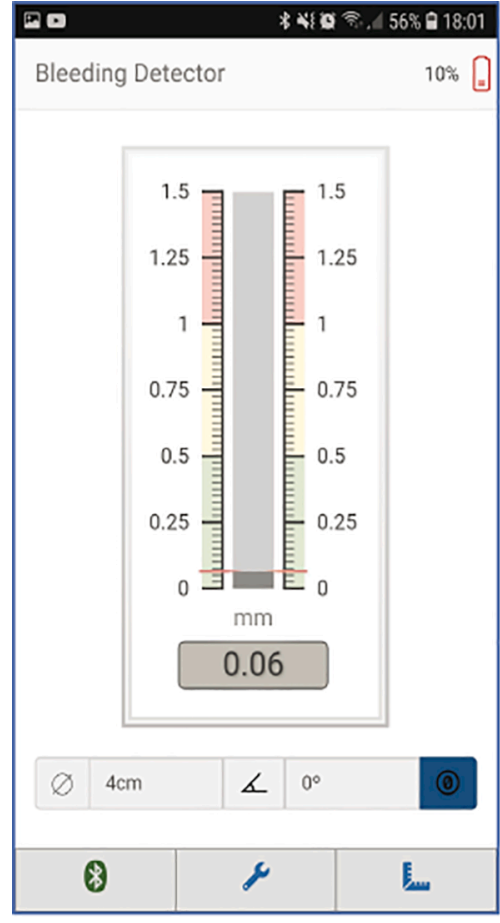

a) light signaling. In both views, at the bottom there are three menu buttons, which allow (from left to right) to connect the application with the microcontroller, to open the configuration view and to open the main view. Once the application is connected to the microcontroller, the values are automatically read and updated every second. At that time, at the top, the supercapacitor charge status indicator also appears.

\subsection{Setup for measuring the detector sensitivity and calibration}

We have used the PMMA instrumented applicator shown in Fig. 4. To perfom the calibration tests we have used water as equivalent fluid. The incorporation of water in the applicator has been carried out using the commercial 7PV1000 pipettor (min. $200 \mu \mathrm{l}, 5 \mu \mathrm{l}$ resolution) from Bibby Sterilin Ltd. Measurements have been made by controlling the room

\begin{tabular}{|c|c|c|c|}
\hline \multicolumn{2}{|l|}{ 国 } & \multicolumn{2}{|c|}{ * 4 (c) ร $.456 \%$ ด 18:01 } \\
\hline \multicolumn{4}{|c|}{ Bleeding Detector } \\
\hline \multicolumn{4}{|c|}{ Tube diameter } \\
\hline$\varnothing$ & $4 \mathrm{~cm}$ & & $\checkmark$ \\
\hline \multicolumn{4}{|c|}{ Color labels configuration } \\
\hline Min & 0.00 & Max & 0,50 \\
\hline Min & 0,50 & Max & 1,00 \\
\hline Min & 1.00 & $\operatorname{Max}$ & 1,50 \\
\hline \multicolumn{4}{|c|}{ Sensor sensibility } \\
\hline \multicolumn{4}{|l|}{0,32} \\
\hline \& & & & س \\
\hline
\end{tabular}

b)

Fig. 5. Mobile application developed. a) Main view; b) Configuration view. 
temperature around $19-22^{\circ} \mathrm{C}$.

Data are collected using the developed monitoring application. Tests were done from $0^{\circ}$ to $45^{\circ}$ tilting angles at 7 different values. For each of these angles, 10 calibrations have been carried out at different orientations ( 8 points were acquired to obtain one calibration process, each point corresponding to the average of 10 measurements). Three different observers have repeated these tests over a period of two months.

In order to compare the laboratory results with the one simulating the most realistic clinical conditions, a specific setup has been considered. It consists of a piece of meat located at the base of the applicator, imitating the irregularities produced by the tumor bed, as shown in Fig. 6. As in clinical practice the base of the applicator would be slightly depressed with respect to the tumor bed (Fig. 6), the detector has been located at a distance of approximately $2 \mathrm{~mm}$ measured from the base of the applicator. This is a zero condition to take into account at the beginning of each measurement, as shown in the developed monitoring application.

\subsection{Setup for measuring the detector performance under radiation}

Three important aspects have been analysed to evaluate the possible alterations caused by the instrumented applicator under radiation conditions: i) sensor operation under and without radiation; ii) possible disturbance of the clinical treatment beam; iii) peripheral SR. Fig. 7 shows the setup used for these measurements. Applicators of $4 \mathrm{~cm}$ and 7 $\mathrm{cm}$ diameter have been used.

i) Sensor operation under and without radiation.

In order to verify whether the detector can work not only in pretreatment mode but also in continuous mode (during irradiation), the behaviour of the system with and without radiation has been evaluated. For this purpose, measurements were taken for different electron energies: the minimum $(6 \mathrm{MeV})$ and maximum $(12 \mathrm{MeV})$ for a $1 \mathrm{~cm}$ fluid height.

ii) Possible disturbance of the clinical treatment beam.

Another aspect evaluated has been the disturbance of the clinical beam. The beam has a significant component of scattered electrons in the applicator, so it is convenient to compare the beam produced by a standard applicator vs the instrumented one. This comparison has been carried out evaluating their main profiles for the two extreme energies (6 and $12 \mathrm{MeV}$ ).

To estimate potential changes in the very low energy component from applicator wall, radichromic films have been used over a solid water phantom, at $1 \mathrm{~cm}$ depth and in contact with the applicator end (0 depth). GAFChromic EBT3 (Ashland Inc., Bridgewater, NJ, USA) film fragments were used. The fragments were handled, scanned and calibrated following the protocol detailed by [14]. The flatbed scanner used was an Epson Expression 12000XL (Seiko Epson Corporation, Nagano, Japan). Doses were obtained with the radiochromic film dosimetry web application Radiochromic.com (Radiochromic SL, Benifaió, Spain). The

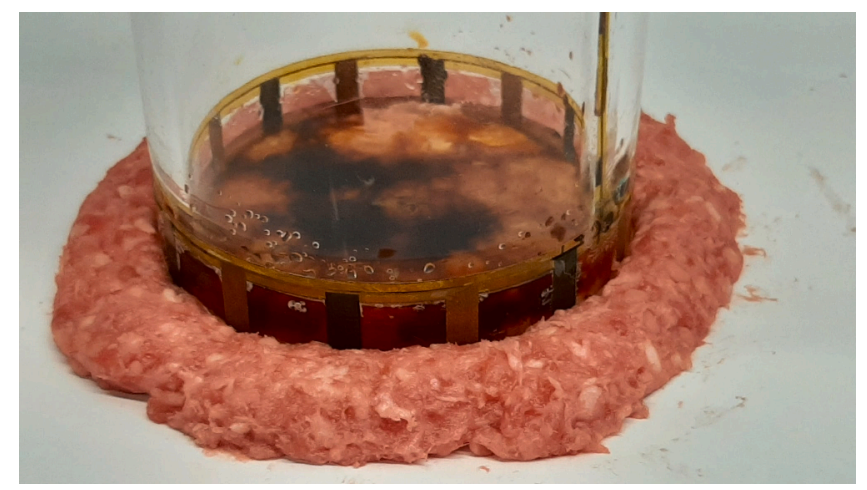

Fig. 6. View of the instrumented applicator with a piece of meat located at its base.

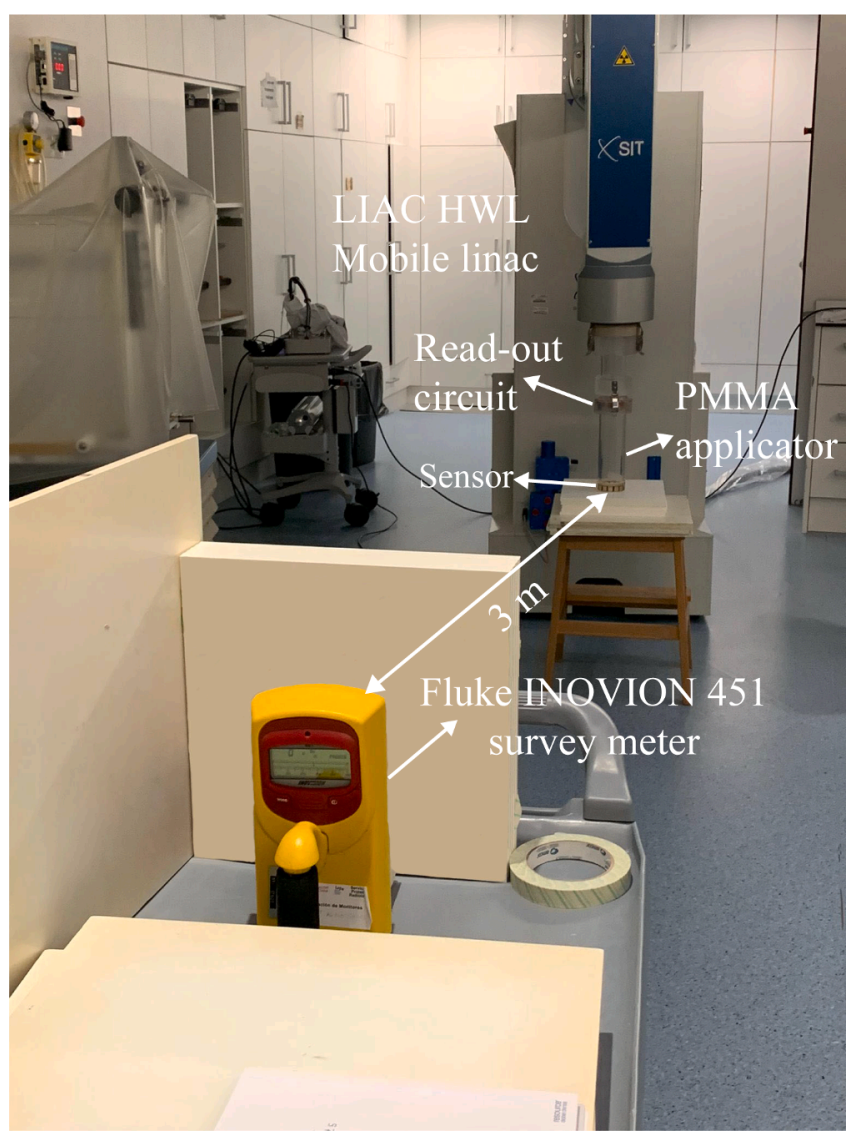

Fig. 7. Experimental setup at La Fe Hospital (Valencia, Spain) for measuring the detector performance under radiation.

uncertainty was estimated as $3 \%$.

iii) Peripheral SR.

As commented above, one of the most important features of the mobile electron linacs is the low SR component, being the x-rays produced by electron bremsstrahlung the main contamination component. The SR with instrumented applicators compared with the standard ones have been also evaluated for the worst-case energy (12 MeV) using the setup described in [10], as shown in Fig. 7. The measurement is performed $3 \mathrm{~m}$ away from the target with a Fluke INOVION 451 survey meter. The procedure is as follows: i) the highest value is taken in the dose rate mode for 300 monitor units at each irradiation; ii) the irradiation is repeated three times for each applicator (standard and instrumented); iii) the ratios between them are obtained. The statistical uncertainty of the ratios was estimated as $2 \%$.

The setup used allows us to establish a minimum standard for the wall of any surgery room [10]. In front of the survey meter a $5 \mathrm{~cm}$ thickness PMMA slab was set, enough for shielding the low energy electrons escaping from the applicator, as typically done in this type of measurements [15].

\section{Results}

\subsection{Sensitivity and calibration results}

Fig. 8 shows the sensitivity (estimated fluid height vs output voltage $V_{o}$ ) for different applicator tilting angles (where $\phi=0^{\circ}$ represents applicator in vertical position). Sensitivity is obtained from a set of calibration curves. These curves in turn have been obtained by representing the output voltage $V_{o}$ as a function of the fluid height, measured on the applicator wall with respect to the horizontal surface.

Sensitivity fit curve as a function of the tilting angle gives $0.070 \bullet \phi+$ 


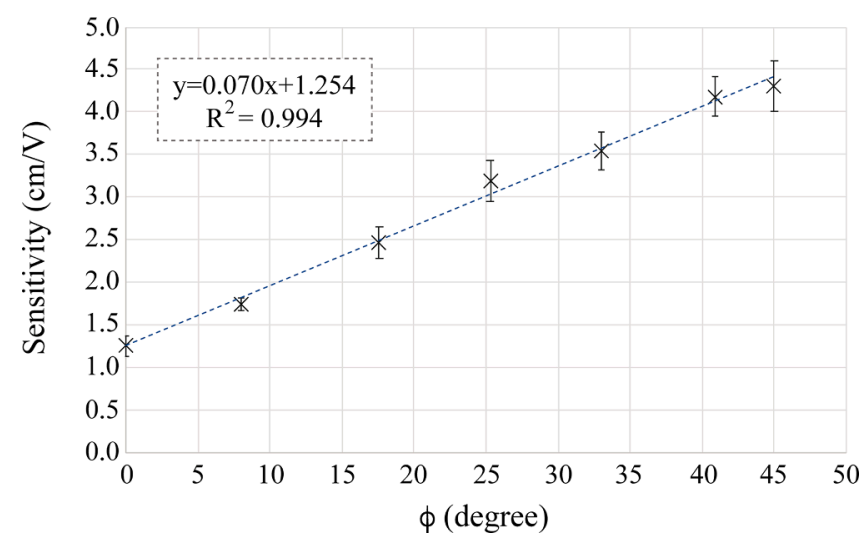

Fig. 8. Sensitivity as a function of the applicator tilting angle $(\phi)$.

1.254. This function is programmed in the mobile application to determine the fluid height in the applicator.

Fig. 9 shows the correlation between the estimated height and the actual height for a set of measurements. Error bars in the height estimation are compatible with those associated with the experimental calibration (Fig. 8).

Fig. 10 shows results obtained with a piece of meat located at the base of the applicator for a $0^{\circ}$ tilting, simulating the irregularities produced by the tumor bed. Measurements have been made after having submerged the piece of meat in water, guaranteeing its hydration. In this case, the height occupied by the piece of meat in the applicator slightly
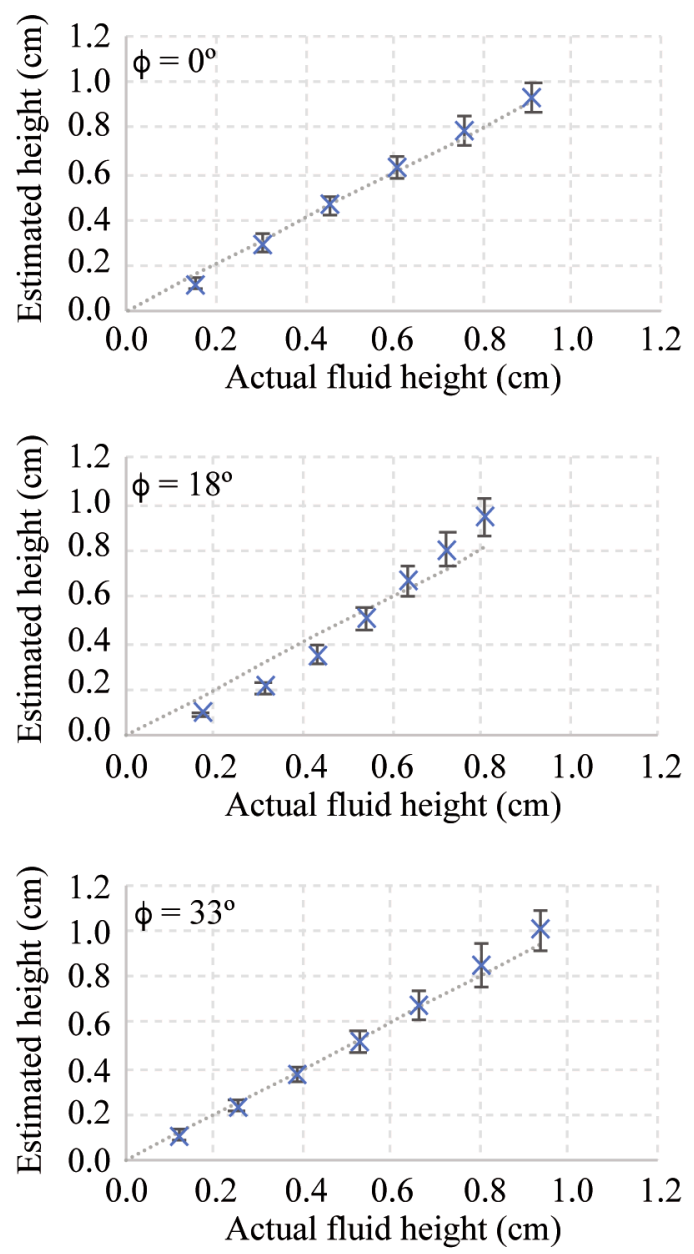

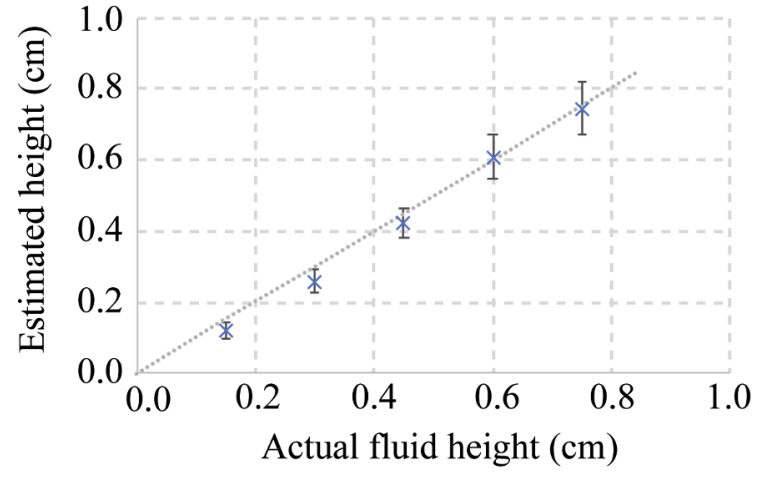

Fig. 10. Estimated fluid height vs actual height at $0^{\circ}$ using a piece of meat at the base of the applicator. Dotted line only represents the ideal response of the detector.

limits the measuring range on the sensor.

\subsection{Detector performance in the linac environment}

No changes were appreciated in the performance of the instrumented applicator-based detector with or without radiation. Estimated height given by the mobile application showed invariability below $4 \%$.

Fig. 11 shows results for $6 \mathrm{MeV}$ and $12 \mathrm{MeV}$ irradiations with comparisons between the dose distributions delivered with both applicators. A global gamma analysis with $2 \% / 2 \mathrm{~mm}$ tolerance and $10 \%$ dose threshold has been performed. Doses were normalized to the central
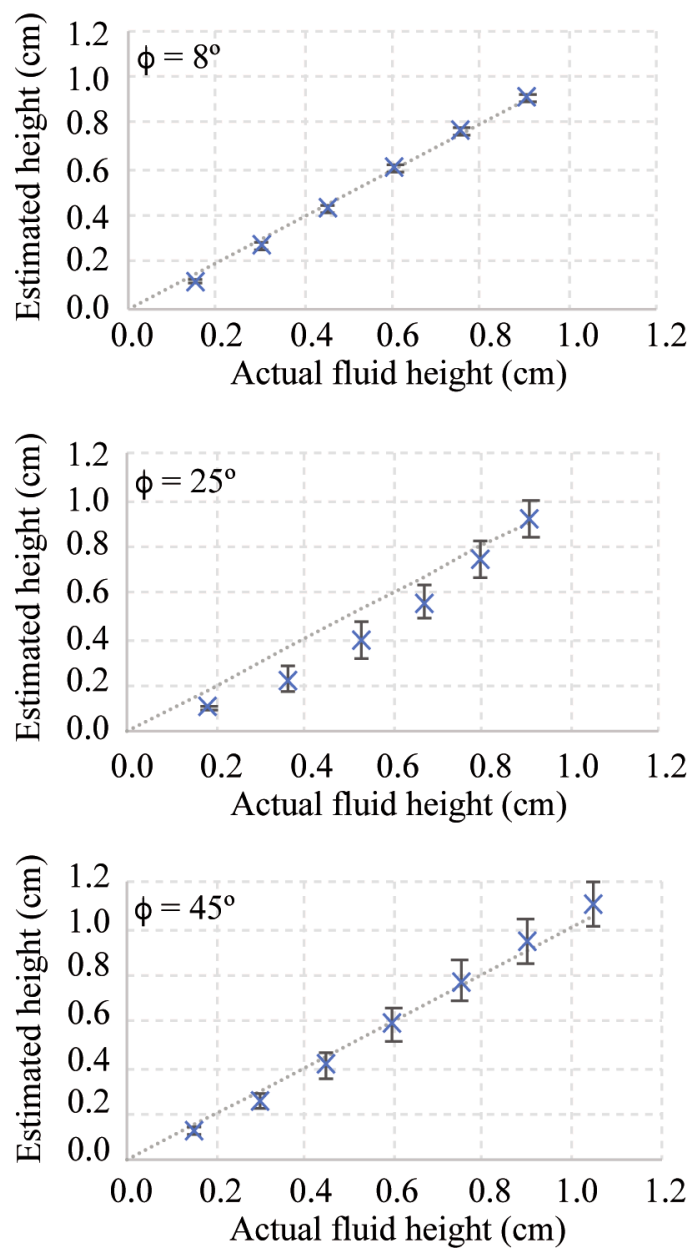

Fig. 9. Estimated fluid height vs actual height for different tilting angles. Dotted line only represents the ideal response of the detector. 

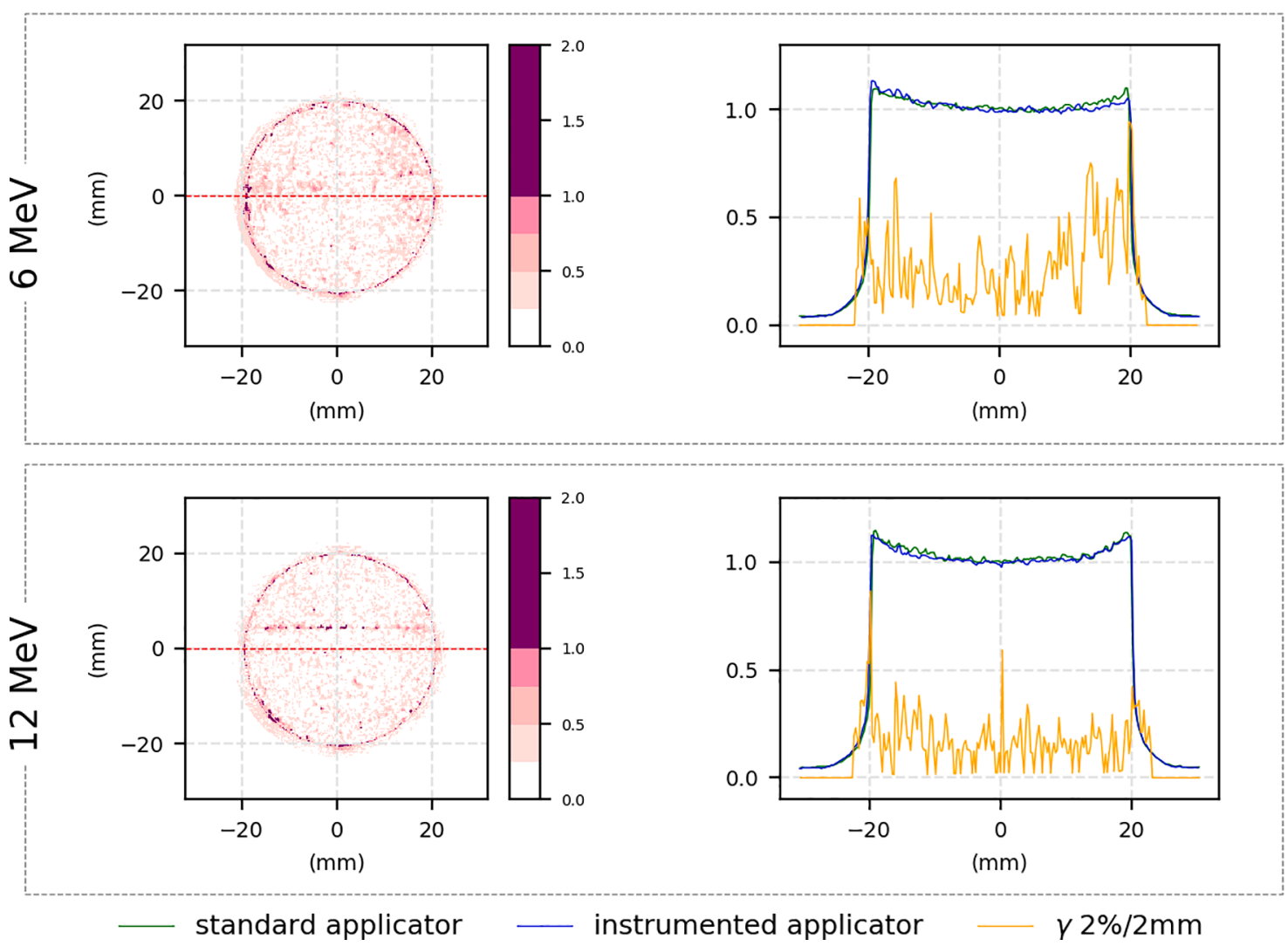

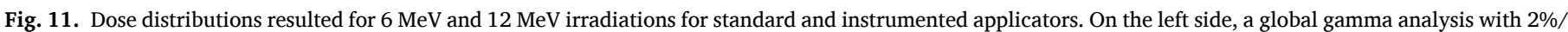

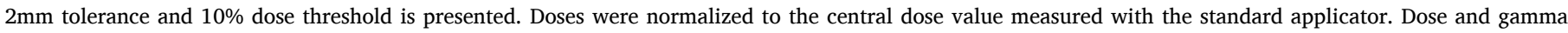

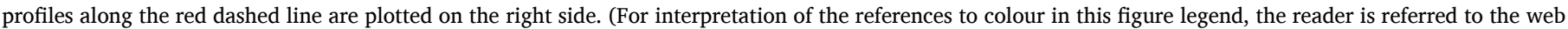
version of this article.)

dose value measured with the standard applicator. The percentage of points with $\gamma$ less than 1 was $99.1 \%$ for both energies and then it is concluded that no dosimetric differences exist.

No SR variations have been found in the measurements made for both standard and instrumented applicators, thus maintaining the excellent SR performance of this linacs.

\section{Discussion}

In order to estimate the fluid height in the applicator, the sensitivity of the system for different tilting angles has been obtained, thus simulating a real irradiation scenario. The sensitivity dependence with the angle has been assumed linear (Fig. 8), obtaining a correlation coefficient $\left(R^{2}\right)$ close to unity (0.994). The maximum error obtained for the sensitivity is $0.295 \mathrm{~cm} / \mathrm{V}$ for $45^{\circ}$ tilting angle. The sensitivity error is around $0.100 \mathrm{~cm} / \mathrm{V}$ for tilting angles of $10^{\circ}$, double for inclinations greater than $15^{\circ}$ and triple for the $45^{\circ}$ extreme case. This increase of the error with the angle is not a big issue from the misadministration dose point of wiew because for tilting angles greather than $20^{\circ}$ the fluid accumulates in a corner of the applicator and not in its central part $[11,12]$.

In accordance with the errors associated to the sensibility (Fig. 8), the estimated height vs the actual height (Fig. 9) also shows a greater divergence between the actual and estimated values as the tilting angle increases. Comparison of estimated fluid height vs real height gives an error of $1 \mathrm{~mm}$ for tilting angles less than $10^{\circ}$ and $2 \mathrm{~mm}$ for those greater than $15^{\circ}$. The minimum detectable fluid height is below $2 \mathrm{~mm}$.

It is important to point out that calibration curves depend both on the applicator diameter and on the detector location and its assembling. Any modification of this circumstance during the manufacturing process would require a new calibration process of the system prior to its sealing for subsequent clinical use.

The result obtained with the setup that simulates the irregularities of the tumor bed (Fig. 10) are within the error margins obtained with the laboratory setup (Fig. 9 at $0^{\circ}$, upper left).

The mobile monitoring application has proven to be very useful for the surgery team due to its simplicity in displaying the results and easy configuration. The traffic light allows the surgery team to quickly decide whether to stop the procedure to remove the accumulated fluid in the applicator.

The behaviour of the detector under radiation conditions $(4 \mathrm{~cm}$ and $7 \mathrm{~cm}$ applicator diameters) has proven its usefulness as a permanent detector since neither its functionality nor its measurements have been altered by radiation (Fig. 10). Besides, because the detector is embedded in the applicator, no disturbance has been observed either in the clinical beam or in the SR around the linac.

According to the measurements we have carried out, the lack of material due to the emptying of the instrumented applicators does not influence either the peripheral radiation at $3 \mathrm{~m}$ or the useful beam. In the hypothetical case that a future clinical application shows an alteration in the parasitic radiation near the applicator, this could easily be modified by filling the free space in its head with the same PMMA material".

\section{Conclusions}

We have presented a low-cost, easy-to-use and ready-to-industrialize permanent bleeding detector integrated in a PMMA applicator for IOERT. It includes a user-friendly mobile application that allows the surgical team to verify the presence of accumulated fluid in the applicator before and during irradiation. 
The proposed solution meets all clinical requirements: i) no contact with patient; ii) enough detection accuracy at any applicator tilting angle; iii) linac-independency; iv) no interference with the irradiation beam.

The proposed detector can be very useful in the clinic, especially in highly-vascularized scenarios where bleeding may cause misadministration dose.

\section{Declaration of Competing Interest}

The authors declare that they have no known competing financial interests or personal relationships that could have appeared to influence the work reported in this paper.

\section{Acknowledgments}

We express our gratitude to V. Carmona, J.C. Ruiz, N. Tejedor, L. Zazo from Department of Radiation Oncology, La Fe Hospital, Valencia, Spain and J. L. Bonet from PTW Iberia S.A.U, Valencia, Spain. Their contributions have been very important during the tests carried-out at La Fe Hospital.

We would like to thank technicians and engineers from IFIC (Centro Mixto UVEG-CSIC, Valencia, Spain) for their help during the embedding of the detector into the applicators.

We also thank S.I.T. SORDINA IORT Technologies SpA for providing us with the applicators used during the tests, within the framework of a previous collaboration established with the University of Valencia. In particular, we express our gratitude to Dr. Giuseppe Felici for his technical help.

\section{References}

[1] Gunderson LL, Willett CG, Intraoperative CFAHL. Irradiation. Techniques and Results. 2th ed. Springer; 2011.

[2] Beddar AS, Biggs PJ, Chang S, Ezzell GA, Faddegon BA, Hensley FW MM. Intraoperative radiation therapy using mobile electron linear accelerators; Report of the AAPM Radiation Therapy Committee Task Group No. 72. Med Phys 2006;12 1476-89.
[3] Calvo FA. Intraoperative irradiation: Precision medicine for quality cancer control promotion. Radiat Oncol 2017;12:1-5. https://doi.org/10.1186/s13014-0170764-5.

[4] Haddock MG. Intraoperative radiation therapy for colon and rectal cancers: A clinical review. Radiat Oncol 2017;12:1-8. https://doi.org/10.1186/s13014-0160752-1.

[5] Roeder F, Krempien R. Intraoperative radiation therapy (IORT) in soft-tissue sarcoma. Radiat Oncol 2017;12:1-13. https://doi.org/10.1186/s13014-016-07512.

[6] Krengli M, Pisani C, Deantonio L, Surico D, Volpe A, Surico N, et al. Intraoperative radiotherapy in gynaecological and genito-urinary malignancies: Focus on endometrial, cervical, renal, bladder and prostate cancers. Radiat Oncol 2017;12 (1). https://doi.org/10.1186/s13014-016-0748-x.

[7] Felici G, Barca P, Barone S, Bortoli E, Borgheresi R, De Stefano S, et al. Transforming an IORT Linac Into a FLASH Research Machine: Procedure and Dosimetric Characterization. Front Phys 2020;8. https://doi.org/10.3389/ fphy.2020.00374.

[8] Vozenin MC, Baumann M, Coppes RP, Bourhis J. FLASH radiotherapy International Workshop. Radiother Oncol 2019;139:1-3. https://doi.org/10.1016/j. radonc.2019.07.020.

[9] López-Tarjuelo J, Morillo-Macías V, Bouché-Babiloni A, Ferrer-Albiach C, SantosSerra A. Defining Action Levels for In Vivo Dosimetry in Intraoperative Electron Radiotherapy. Technol Cancer Res Treat 2016;15(3):453-9. https://doi.org/ $10.1177 / 1533034615588196$

[10] Sanchis E, Casans S, Felici G, García-Gil R, Sanchis-Sánchez E, Pérez-Calatayud I, et al. Detector for monitoring potential bleeding during electron intraoperative radiotherapy. Phys Medica 2019;57:95-9. https://doi.org/10.1016/j. ejmp.2018.12.010.

[11] Sanchis E, Casans S, García-Gil R, Martos J, Sanchis-Sánchez E, Pérez-Calatayud I, et al. Improving bleeding detector features for electron intraoperative radiotherapy. Phys Medica 2019;65:150-6. https://doi.org/10.1016/j ejmp.2019.08.015.

[12] Garcia-Gil R, Casans Berga S, Sanchis-Sanchez E, Perez-Calatayud I, PerezCalatayud J, Sanchis Peris E. Electronic design for a bleeding detector to be used in intraoperative radiotherapy applications. IEEE Sens J 2021;21(4):4786-92. https://doi.org/10.1109/JSEN.2020.3032828.

[13] Sordina IORT Technologies. LIACHWL Mobile IOeRT Accelerator s. f. https:// www.vertec.co.uk/content/uploaded//LIAC HWL.pdf (accedido 4 de diciembre de 2019).

[14] Méndez I, Rovira-Escutia JJ, Casar B. A protocol for accurate radiochromic film dosimetry using Radiochromic.com. Radiol. Oncol 2021;55:369-78. https://doi, org/10.2478/raon-2021-0034.

[15] Soriani A, Felici G, Fantini M, Paolucci M, Borla O, Evangelisti G, et al. Radiation protection measurements around a $12 \mathrm{MeV}$ mobile dedicated IORT accelerator. Med Phys 2010;37(3):995-1003. https://doi.org/10.1118/1.3298012. 